\title{
Publicaciones racionales o emocionales en comunidades de marca en Facebook - El caso «Starbucks México»
}

\section{Rational or emotional posts on Facebook brand communities - The Mexico Starbucks Case}

\begin{abstract}
Luceli Karina Ponce es maestra en comunicación de la Universidad de Quebec en Montreal (UQAM, Canadá) (luceli2@gmail. com) (https://orcid.org/0000-0003-4458-4207)

Dr. Benoit Cordelier es profesor e investigador en la Universidad de Quebec en Montreal (UQAM, Canadá) (cordelier.benoit@ uqam.ca) (http://orcid.org/0000-0002-7389-0353)
\end{abstract}

\begin{abstract}
Resumen
El propósito de este artículo es demostrar que las emociones son elementos esenciales en la participación entre los miembros de una comunidad de marca en un sitio de redes sociales. Estudiamos las expresiones emocionales dentro de una comunidad de marca en línea con una metodología mixta basada en un análisis de contenido con dos codificadores de la misma cultura de origen. Analizamos 77 publicaciones y 13043 comentarios de miembros de la comunidad de la marca "Starbucks México» en Facebook, publicados entre enero y junio de 2014. Se descubrió que las personas participan más a través de emociones relacionadas con la felicidad, como el amor, pasión y deseo. Este documento incluye el estudio de emociones positivas y negativas expresadas en una comunidad de marca en Facebook. Además, se expone el nivel de participación que generan las emociones, así como la distinción de los elementos emocionales aplicados en la comunicación entre una empresa y los miembros de una comunidad de marca. Categorizamos las publicaciones de la marca teniendo en cuenta las dimesiones emocionales y racionales-promocionales. En definitiva, este trabajo contribuye a la literatura al señalar que más que la expresión de emociones por sí sola, es la combinación de elementos emocionales y promocionales de la página de marca lo que desencadena el volumen de comunicación.
\end{abstract}

\begin{abstract}
The aim of this article is to demonstrate that emotions are essential elements of participation between members of a brand community in a social networking site. Emotional expressions were studied within an online brand community through a mixed method approach based on a content analysis with two coders of the same cultural origin. 77 posts and 13,043 comments were analyzed from members of the brand community "Starbucks Mexico" on Facebook, reported between January and June 2014. It was found that people participate more in the presence of hapiness related emotions such as love, passion, and desire. This paper is interested in positive and negative emotions expressed in a Brand Community on Facebook. Additionally, the level of participation that emotions generate is presented, as well as the distinction of emotional elements applied in the communication between a company and the members of a brand community. This paper contributes to the literature by pointing out that more than the expression of emotions, it is the combination of emotional and promotional elements from the brand page that triggers more communication volume.
\end{abstract}

\section{Palabras clave I keywords}

Emociones, comunicación promocional, comunidad de marca, participación, Facebook.

Emotions, promotional communication, brand community, participation, Facebook.

Cómo citar: Ponce, L.K. y Cordelier, B. (2019). Publicaciones racionales o emocionales en comunidades de marca en Facebook - El caso «Starbucks México». Retos Revista de Ciencias de la Administración y Economía, 9(17), 109-126. https://doi.org/10.17163/ret.n17.2019.07 


\section{Introducción}

La evolución de la sociedad en nuestra vida diaria incluye el desarrollo de redes sociales en Internet, así como la construcción de comunidades de marca. La penetración de internet en México es una de las más altas en América Latina y sigue creciendo: 53.0 millones de usuarios (2014), 65.8 millones (2015), 70 millones (2016) y 79.1 (2017), que representa un 67\% de la población de personas mayores a 6 años en 2017 (Asociación de internet.MX, 2018). Además en México, Facebook sigue siendo la principal red social con un alcance del 98\% de los usuarios (Asociación de internet.MX, 2018).

Dentro de las redes sociales, la interacción se refuerza entre los miembros de dichas comunidades fomentando directamente las emociones (Hollebeek, Juric, \& Tang, 2017; Shankar, Elliott, \& Fitchett, 2009). Dentro de una comunidad de marca, los afiliados son una fuente importante de información (Firat \& Venkatesh, 1995). Esto significa que el contenido generado por sus miembros puede representar una ventaja importante para las empresas, ya que sus comentarios arrojan información sobre el éxito y la aceptación de productos o servicios específicos y que mejoran la relaciones con los consumidores (Weiger, Hammerschmidt, \& Wetzel, 2018).

En el caso de las redes sociales, otras investigaciones sugieren que éstas se han convertido en una «etapa» (Cordelier \& Turcin, 2005) donde los usuarios expresan emociones a diario (Barroso Morales, 2011). Esta narrativa emocional del "yo» en forma de textos e imágenes permite a los usuarios crear su espacio virtual, donde expresan sus emociones y mantienen un contacto cercano con las historias de otros miembros, basadas en la mediación tecnológica. Las emociones favorecen un desarrollo social (Evans, 2002; Plutchik, 1994; Yu, 2014) que puede beneficiar estrategias de branding (Roberts, 2005; Yu, 2014).

Por lo tanto, el propósito de la presente investigación no es tanto relacionar directamente beneficios económicos con la participación de los internautas, sino más bien centrarse en el tipo de comunicaciones que generan más participación, comprobar que la comunicación emocional es más importante y proponer una categorización de las publicaciones de la marca. En otras palabras, nuestro objetivo es detectar las emociones positivas o negativas expresadas en Facebook, así como relacionarlas con el nivel de participación generado por el tipo o estilo de comunicación de las mismas.

Nos hemos interesado en Starbucks por ser una importante lovemark, según la expresión popularizada por el publicitario Kevin Roberts (2005), que en poco tiempo pasó a dominar el mercado de las cafeterías en México ${ }^{1}$ y que está dentro de las más importante en Facebook con más de 4 millones de likes.

Después de una revisión de literatura sobre emociones y comunidades de marca en línea, presentamos nuestra metodología basada en un análisis de contenido antes de analizar y discutir las categorías de publicación más interesantes para generar participación.

1 En septiembre del 2002 abre su primera sucursal en la ciudad de México. A finales del 2016, tenía una participación en el sector, por número de unidades, de 39\%, seguido por Italian Coffee Company con 11\% y Café Punta del Cielo con 10\%, según datos de Euromonitor (Expansión, 2017). 


\section{Emociones y comunidades de marca en línea}

\subsection{Teoría de las emociones}

Existen varias perspectivas teóricas sobre emociones ya sean psicológicas, psicosociales, filosóficas o antropológicas (v. gr. Evans, 2002; Huang, 2001; Illouz, 2007; Le Breton, 2012). Esta investigación se basa principalmente en las emociones básicas de Robert Plutchik (1994, 2001).

El autor (ob. cit) identificó cuatro emociones básicas, que integró en pares opuestos: alegría vs tristeza; confianza vs disgusto; miedo vs ira y anticipación vs sorpresa. Según Plutchik (ob. cit), estas emociones «básicas» son biológicamente primitivas. También las describe como adaptaciones básicas que necesitan todos los organismos en la lucha por la supervivencia individual. Por lo tanto, las emociones tienen una estrecha relación con los procesos biológicos adaptativos. Su teoría de la emoción sugiere que no son solo elementos, sino también circunstancias que comienzan con un estímulo que provoca sentimientos, cambios psicológicos, acciones y un comportamiento orientado hacia algún objetivo (Plutchik, 2001).

Las emociones juegan un papel importante en la toma de decisiones humanas. El tono y el contenido utilizado en la comunicación en las redes sociales tienen un impacto considerable en las personas, ya que las campañas positivas llegan a una mayor cantidad de clientes. La conducta expresiva humana que comunica una emoción adquiere ciertas propiedades fijas que pueden reconocerse independientemente de la cultura y el aprendizaje, a pesar de ciertas diferencias en su interpretación (Buck, 2006; Burgoon, Guerrero, \& Floyd, 2010; Plutchik, 2001).

Otros estudios muestran que las emociones están relacionadas con los procesos evolutivos que han mantenido a los humanos a salvo durante siglos. Por ejemplo, la ira y el miedo son muy primitivos dentro del comportamiento humano y su función es estar preparados para prestar atención (Hochschild, 1979; Plutchik, 1994, 2001). Nuestras emociones también están vinculadas biológicamente con el sentido, ayudan a comprender nuestra relación con los demás y son esenciales para la socialización (Hochschild, 1979).

Por otro lado, también estudiamos las emociones secundarias con el fin de lograr una escala de datos más amplia. Estas se componen combinando las primarias. Aquí algunos ejemplos, pero hay muchos más: irritabilidad, hostilidad, dolor, melancolía, soledad, ansiedad, miedo, pánico, disfrute, felicidad, confianza, amabilidad, afecto, amor, conmoción, asombro, aversión, disgusto, culpa, vergüenza, arrepentimiento (Buck, 2006; Evans, 2002; Frederickson, 2017; Plutchik, 2001).

Las emociones se pueden dividir en positivas y negativas. Las positivas, como la felicidad, aumentan los recursos físicos, sociales e intelectuales, construyen reservas emocionales ante un desafío. Las emociones negativas como el miedo, la tristeza y la ira son defensas naturales contra las amenazas externas (Buck, 2006; Plutchik, 2001). Por tanto, las emociones rigen los patrones de pensamiento humano, las creencias, los comportamientos, las actitudes y las respuestas a las experiencias de la vida siendo también críticas en el proceso de socialización (Le Breton, 2012). 


\subsection{Emociones, marketing y redes sociales}

Las emociones, dentro del marketing y la publicidad, contribuyen al desarrollo de mensajes y estrategias de comunicación. Los profesionales de la publicidad también desarrollan un amplio interés por el desempeño de las emociones y su potencial social. En palabras del publicista Kevin Roberts (2005, p. 44), influenciado por el trabajo de Dylan Evans (v. gr. Evans, 2002):

Para mí, lo realmente sorprendente de las emociones secundarias es lo sociales que son y lo importantes que son. Puedes sentir emociones primarias cuando estás solo. Pero para desarrollar una emoción secundaria necesitas estar con alguien más.

Las emociones juegan un papel importante en la comprensión de un mensaje y son elementos esenciales de la interacción, ya que generan la participación dentro de las comunidades de marca en las redes sociales. No obstante, falta investigación sobre el tema en relación con la comunicación persuasiva y los medios, incluyendo internet y la comunidades de marca en línea (Hollebeek et al., 2017; Nabi, 2017). En palabras de Nabi (2017, s.p.):

Si bien la historia del estudio de la persuasión en los medios de comunicación es larga y robusta, el examen de la emoción en tales procesos es más limitado. Sin embargo, se sigue prestando atención al papel de muchas emociones, incluido el miedo, la culpa y el humor, en el proceso de mensajes persuasivos en los medios. Aunque menos centradas en las emociones, tendencias recientes en la investigación sobre narrativas persuasivas y mensajes virales sugieren que la emoción puede jugar un papel clave en la persuasión basada en los medios.

Dentro de las ventajas del estudio de la emociones encontramos que al generar mensajes que coinciden con las emociones positivas, las empresas pueden establecer y mejorar su conexión con su público o consumidores meta (Codina, Rodríguez, \& Cadena, 2017; Guerreiro, Rita, \& Trigueiros, 2015; Khuong \& Tram, 2015; Weiger et al., 2018) ya que los consumidores se animan más con las publicaciones de tipo social (Yu, 2014). Los beneficios económicos se basan también en un refuerzo de los vínculos sociales que así se producen y que Manchanda, Packard y Pattabhiramaiah (2015) llaman «dólares sociales» (social dollars).

Si bien se pueden seguir lamentando lagunas (Nabi, 2017), el tema de las emociones va ganado popularidad entre los académicos y se pueden encontrar una cantidad considerable de artículos relacionados con este tema. Por ejemplo, los estudios muestran que dentro de las comunidades de marca, campo de interés científico, los individuos crean vínculos de identificación con las empresas y con otros miembros de las comunidades a través de interacciones emocionales (Goulding, 2013; Hudson, Roth, Madden, \& Hudson, 2015; Thompson, Rindfleisch, \& Arsel, 2006; Vincent \& Fortunati, 2009).

También muestran que los consumidores están organizados dentro de esas comunidades para compartir experiencias de una marca (Muñiz \& O'Guinn, 2001; Schembri \& Latimer, 2016). Otros estudios sugieren que la interacción entre los miembros y las empresas se promueve dentro de las comunidades de marcas en línea, lo que a su vez favorece la participación de sus miembros (Schau, Muñiz, \& Arnould, 
2009). Según Ashforth y Mael (1989) para lograr la interacción, los afiliados deben identificarse entre sí, lo que es posible a través de un proceso de socialización que contribuye al proceso de elaboración de la cultura de marca (Schembri \& Latimer, 2016), siendo la emoción un elemento importante en la motivación de los miembros (Dessart, Veloutsou, \& Morgan-Thomas, 2015).

Por su parte, las emociones universales tienen la ventaja de ser aplicadas en diferentes culturas. La facilidad y eficiencia de su aplicación son comparables a las emociones verbales con un grado diferente de intensidad, lo que da como resultado emociones secundarias como el deseo y la pasión entre muchos otros. Por ejemplo, ciertos autores se refieren al deseo como parte de la naturaleza humana, que es una de las fuerzas que desencadenan un comportamiento (Buck, 2006; Pell, Monetta, Paulmann, \& Kotz, 2009; Plutchik, 2001). El deseo es un motivo para satisfacer necesidades o sentimientos (Baun \& Gröppel-Klein, 2003; Belk, Ger, \& Askegaard, 2003). Se encuentra a menudo en la publicidad y el marketing, ya que es un motor de acción que expresa una necesidad a través del lenguaje y genera interés y participación (Goulding, Shankar, Elliott, \& Canniford, 2009). Al activar un deseo, la marca genera cercanía con los consumidores y logra una experiencia personal con el producto; es una necesidad al principio, pero se convierte en un deseo cuando las personas piensan sobre un producto en particular (Belk et al., 2003).

Otros estudios sobre las emociones proponen que la felicidad es contagiosa y se propaga más rápido en las redes sociales (Kramer, Guillory, \& Hancock, 2014). También argumentan que las personas felices reúnen más seguidores en las redes sociales y otros grupos sociales. Los estados emocionales pueden transferirse a otros a través del contagio emocional, lo que lleva a las personas a experimentar inconscientemente las mismas emociones (Berger \& Milkman, 2012; Coviello et al., 2014; Fowler \& Christakis, 2008). Además, las emociones expresadas en redes digitales influyen en nuestros propios sentimientos y son evidencia de un contagio emocional a mayor escala, a través de los medios sociales (Brady, Wills, Jost, Tucker, \& Van Bavel, 2017; Cohen, 2014). El contagio emocional se produce a través de la comunicación textual mediada por dispositivos informáticos y permanece incluso, algunos días después de una publicación (Kramer et al., 2014).

Además, con la llegada de las redes sociales, los consumidores comparten contenido en línea y su transmisión influye en la compra de productos, dando resultados colectivos especialmente cuando existe una emoción positiva en el mensaje (Berger \& Milkman, 2012; Fu, Wu, \& Cho, 2017).

En las redes sociales, las emociones se identifican en un nivel no verbal, y los elementos emocionales se pueden analizar en los comentarios del consumidor, como por ejemplo, la presencia de expresiones onomatopéyicas, signos de puntuación, intersecciones, imágenes fotográficas y emoticones (Carvalho, Sarmento, Silva, \& de Oliveira, 2009). La distinción entre «verbal» y «no verbal» es más compleja en la comunicación escrita que en las redes sociales. Además, el desencadenamiento de las emociones está relacionado con la velocidad a la que recibimos y respondemos a la información (Coviello et al., 2014; Scolari, 2013). Dependiendo de la situación, los usuarios influyen directamente en la información de acceso, produciendo participación continua, lo que resulta en más producción y reproducción de contenido. Lo que 
interesa al desarrollo de la comunicación de marketing, ya que la emoción participa en el involucramiento de los consumidores a través de uno de los mecanismos de la economía de la atención (Aguado-Guadalupe, 2017; Auladell, 2016; Ribes, Monclús, Gutiérrez García, \& Martí, 2017; Segarra-Saavedra \& Tur-Viñes, 2017).

\section{Materiales y método}

La presente investigación tiene por objetivo detectar las emociones positivas o negativas expresadas en Facebook y relacionarlas con el nivel de participación generado por el tipo o estilo de comunicación de las mismas. Para conseguirlo, esta investigación intenta responder las siguientes preguntas de investigación: en línea?

RQ1. - ¿Qué tipo de expresiones emocionales se observan en una comunidad

RQ2. - ¿Ayuda la expresión de emociones positivas a generar un mayor volumen de comunicación que la de las negativas y los mensajes más racionales?

\subsection{Muestreo}

Se recopilaron datos cualitativos en una primera fase para sondear la tipología emocional (Buck, 2006; Hupp, Gröppel-Klein, Dieckmann, Broeckelmann, \& Walter, 2008; Plutchik, 2001) y evaluar su relevancia para analizar los comentarios emocionales de los miembros de la comunidad de la marca. El análisis se centró en los comentarios expresados por los miembros de la comunidad de marca «Starbucks» con contenido emocional.

La estrategia de medios sociales de Starbucks podría caracterizarse como bastante proactiva y positiva. La compañía, más que participar, genera conversaciones a través de sus publicaciones en su página de Facebook (fan page). En total se analizaron integramente las 77 publicaciones de la marca Community Starbucks México en Facebook y los 13.043 comentarios generados por sus miembros entre enero y junio de 2014 . El periodo se escogió para incluir diferentes periodos comerciales importantes en la sociedad mexicana. En este intervalo observamos diferentes categorías de información y elementos emocionales expresados por los consumidores. Los datos analizados incluyen eventos propensos a la expresión emocional dentro de los cuales se destacan enero (Año Nuevo y Reyes), febrero (Día de San Valentín) y mayo (Día de la Madre).

\subsection{Análisis de contenido}

El presente estudios analiza las reacciones de los internautas ante las publicaciones de una página de Facebook a través de un análisis de contenido. Este tipo de metodología recopila muchos métodos en sí misma, que permiten categorizar el contenido de las comunicaciones de manera estándar y sistemática (Berelson, 1952; Kassarjian, 1977; Kolbe \& Albanese, 1997; Neuendorf, 2017).

Ha sido utilizado por investigadores interesados en examinar comunicaciones tales como publicidad, historias de medios y sitios web (Neuendorf, 2017). Este método ayuda a lograr una categorización sistemática y objetiva de las emociones siguiendo la taxonomía de Plutchik (2001) y su posible influencia en los flujos de comunicación. Después de este paso realizamos un análisis léxico, en el que se define 
el sentido emocional expresado en las palabras de los comentarios, principalmente enfocado en adjetivos y verbos.

Asímismo se utilizó el método de análisis de sentimiento, también llamado «minería de opiniones», que analiza las opiniones, sentimientos, evaluaciones, actitudes y emociones de las personas con respecto a productos, servicios, organizaciones, individuos, problemas y eventos. (Yadollahi, Shahraki, \& Zaiane, 2017). Con el crecimiento de las redes sociales (por ejemplo, revisiones, discusiones en foros, blogs, microblogs y sitios de redes sociales) se usa el contenido en estos medios como generadores de decisiones y comunicación. Los indicadores más importantes de sentimientos son palabras que se usan comúnmente para expresar sentimientos positivos o negativos. Por ejemplo, «bueno», «maravilloso» e «increíble», son las palabras de sentimiento positivo, y por su parte «malo», "pobre»y «terrible» son claramente palabras de sentimiento negativo (Liu, 2012).

Las expresiones se evaluaron según emociones básicas y secundarias basadas en las emociones primarias y secundarias de Plutchick (2001). Después se creó una tipología de expresiones emocionales con palabras significativas expresadas en español (tabla 1), por ejemplo adjetivos, sustantivos, verbos en base al Diccionario de la Real Academia de la Lengua Española, en su versión online.

Tabla 1. Expresiones emocionales en español

\begin{tabular}{|l|l|l|l|l|l|}
\hline \multicolumn{1}{|c|}{ Amor } & \multicolumn{1}{|c|}{ Placer } & \multicolumn{1}{|c|}{ Deseo } & \multicolumn{1}{|c|}{ Alegría } & \multicolumn{1}{|c|}{ Frustración } & \multicolumn{1}{c|}{ Disgusto } \\
\hline Amar & Encantar & Querer & Felicidad & Tristeza & Feo \\
\hline Besos & Gustar & Desear & Bien & Rabia & Horrible \\
\hline Corazón & Disfrutar & Antojo & Buen & Mal & Asco \\
\hline Vida & Gozar & Ir & Momento & Feo & Malo \\
\hline Pasión & Delicioso & Hacer & Especial & Decepción & Pésimo \\
\hline Junto & Bueno & Necesitar & Mejor & Odiar & Decepción \\
\hline Adorar & Rico & Urgir & Alegrar & Enojo & Desagradable \\
\hline Favorito & Excelente & Probar & Relajar & Nostalgia & Porquería \\
\hline
\end{tabular}

La tipología anterior contiene algunas palabras tomadas como nodos para nuestra codificación y fue verificada con el resultado de los datos generados por el software de análisis de datos cualitativos NVivo, destacando las palabras más frecuentes que se expresan en los comentarios del consumidor. Notamos que la mayoría de las expresiones encontradas por el programa mencionado, corresponden con las palabras incluidas en nuestra tipología. 
Figura 1. Nube de «tags» de palabras frecuentes

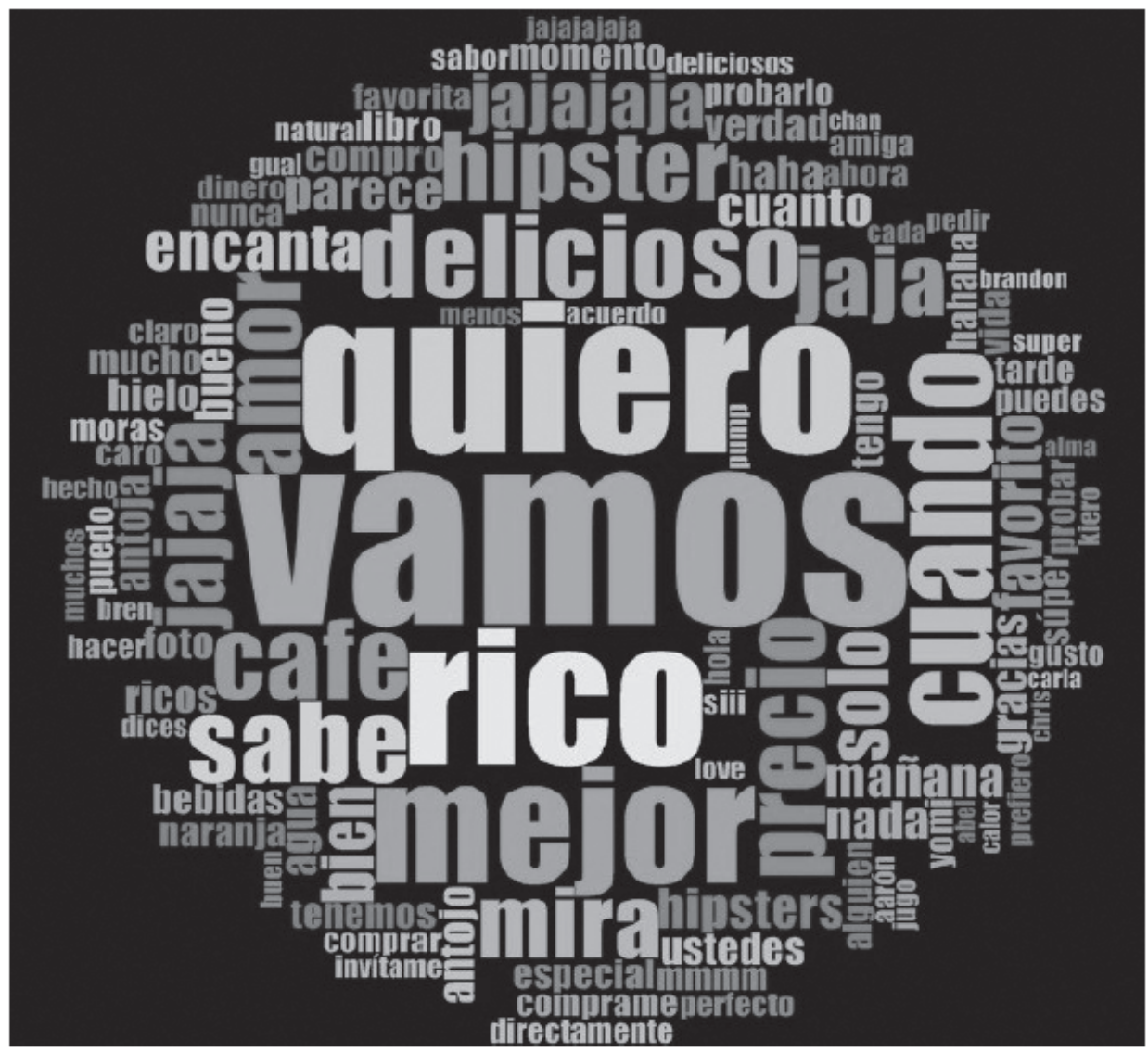

Para distinguir y medir las emociones expresadas por los miembros de la comunidad de la marca, las dividimos en positivas y negativas según las categorías que sobresalieron en la revisión de literatura. Las palabras positivas más comunes encontradas en los comentarios, según nuestra tipología y los resultados de NVivo fueron: vamos, quiero, rico, delicioso, momento, amor, mejor, antojo.

Las palabras negativas encontradas fueron: precio, caro y horrible. Además, identificamos ciertos adverbios cuando las personas expresaban un comentario negativo. Cabe notar que estas palabras surgen más en relación con elementos racionales centrados en el servicio, el precio, la salud y la calidad.

Luego, las variables fueron seleccionadas con base en las emociones universales tales como la felicidad, la tristeza, la sorpresa y la ira (Buck, 2006; Plutchik, 2001). En consecuencia, integramos algunas de las emociones secundarias para identificar mejor las expresiones emocionales tales como la alegría, el amor, la pasión, el deseo, la ansiedad, la frustración y el disgusto (Buck, 2006). 
Tabla 2. Emociones básicas y secundarias

\begin{tabular}{|l|l|}
\hline \multicolumn{1}{|c|}{ Emociones básicas } & \multicolumn{1}{c|}{ Emociones secundarias } \\
\hline Alegría & Amor, pasión, deseo, alegría \\
\hline Tristeza & Ansiedad, nostalgia \\
\hline Sorpresa & Asombro \\
\hline Enojo & Frustración, disgusto \\
\hline
\end{tabular}

Fuente: Elaboración propia basada en las emociones universales y secundarias de Plutchick (2001) y Buck (2006).

Con la ayuda del programa de análisis NVivo, fue posible estudiar cada publicación y comentar para clasificar el contenido en función de las emociones.

\subsection{Fiabilidad}

Se realizó una doble codificación con el objetivo de comprobar la relevancia de la interpretación de las palabras y los emoticones con contenido emocional predominantes en la investigación. Estos concordaban con las características culturales del primer codificador.

La segunda codificación fue realizada sobre el $10 \%$ de la muestra por una persona que comparte la nacionalidad mexicana, el idioma materno (español), el origen étnico, la educación, las tradiciones y los patrones culturales practicados por el investigador principal y los miembros de la comunidad de la marca. La confiabilidad se comprobó a través de la prueba del coeficiente Kappa ( $k$ ) de Cohen (tabla 3), un análisis estadístico que toma también en cuenta el nivel de acuerdo que podría esperarse a través del azar (Fleiss y Cohen, 1973; Landis y Kosh, 1977). Esto se refleja en los resultados calculados con la fiabilidad del coeficiente Kappa ( $k$ ) de NVivo (tabla 4).

Tabla 3. Nivel de Concordancia de la doble codificación

\begin{tabular}{|l|c|c|}
\cline { 2 - 3 } \multicolumn{1}{c|}{} & Coeficiente Kappa & Concordancia \\
\hline Comentarios positivos & 0.62 & $0.40-0.75 /$ Buena \\
\hline Comentarios Negativos & 0.69 & $0.40-0.75 /$ Buena \\
\hline Comentarios Racionales & 0.57 & $0.40-0.75 /$ Buena \\
\hline
\end{tabular}

Tabla 4. Coeficiente Kappa de Cohen por patrón de sentimiento

\begin{tabular}{|l|c|c|}
\cline { 2 - 3 } \multicolumn{1}{c|}{} & Coeficiente Kappa & Concordancia \\
\hline Deseo & 0.6 & $0.40-0.75 /$ Buena \\
\hline Amor & 0.59 & $0.40-0.75 /$ Buena \\
\hline Alegría & 0.32 & $<0.40 /$ Pobre \\
\hline Placer & 0.65 & $0.40-0.75 /$ Buena \\
\hline Desagrado & 0.69 & $0.40-0.75 /$ Buena \\
\hline Ira & 0.64 & $0.40-0.75 /$ Buena \\
\hline Tristeza & 0.58 & $0.40-0.75 /$ Bueno \\
\hline
\end{tabular}


Al codificar los resultados, confirmamos que los elementos emocionales estaban presentes en los comentarios expresados por los miembros de la comunidad de la marca. Los elementos emocionales coincidieron con el 60\% de la información analizada por los codificadores.

\section{Análisis y resultados}

\subsection{Emociones positivas}

Se observó que las emociones positivas están presentes en la interacción entre los miembros de la comunidad de marca, con una fuerte inclinación hacia la felicidad, expresada a través de las emociones secundarias como el deseo, la pasión, el amor, la sorpresa y la alegría.

Esto señala una clara inclinación hacia el deseo y la pasión. Los comentarios positivos también incluyen recomendaciones de productos y la defensa de la marca contra las quejas de otros consumidores. Además, se detectó que el deseo promueve una acción. Se observó también que las expresiones de deseo son seguidas por verbos que indican una acción, por ejemplo: quiero uno (deseo), vamos a probarlo (acción).

Además, la pasión incita a las personas a hacer cosas impulsivamente y relevantes para el momento presente; con frecuencia se expresa a través de adjetivos, como por ejemplo: me encanta (pasión), soy feliz (pasión), me encantan esos momentos (amor, felicidad, pasión).

El deseo y la pasión son las familias de códigos más recurrentes que se encuentran en los datos, seguidas por la alegría, el amor y la sorpresa (tabla 5).

Tabla 5. emociones secundarias positivas comunes

\begin{tabular}{|l|c|}
\multicolumn{1}{|c|}{ Emoción } & Porcentaje \\
\hline Alegría & $21,10 \%$ \\
\hline Deseo & $32,70 \%$ \\
\hline Placer & $30,70 \%$ \\
\hline Amor & $11,00 \%$ \\
\hline Sorpresa & $4,50 \%$ \\
\hline
\end{tabular}

\subsection{Emociones negativas}

Las emociones negativas observadas en el estudio se expresaron a través de la frustración, el disgusto y la nostalgia (tabla 6). Sin embargo, estos elementos también activan la interacción y la participación dentro de las comunidades de marca. Además, se percibió que algunas de las expresiones negativas se formularon sobre cuestiones racionales como el precio, la salud, la dieta y la calidad. 
Tabla 6. Emociones secundarias negativas comunes

\begin{tabular}{|l|c|}
\multicolumn{1}{|c|}{ Emoción } & Porcentaje \\
\hline Frustración & $29,25 \%$ \\
\hline Disgusto & $60,00 \%$ \\
\hline Nostalgia & $10,75 \%$ \\
\hline
\end{tabular}

\subsection{Comentarios por categoría de publicación}

Al comienzo de la investigación nuestro objetivo era identificar la presencia del producto, así como la existencia de actividad promocional y la presencia de elementos informativos que llamamos «racionales».

Luego distribuimos las publicaciones hechas por la marca en las siguientes categorías de mensajes:

- Promocional con elementos emocionales: la marca publica un mensaje que incluye una promoción, usando frases o imágenes emocionales. Por ejemplo: "Prueba el nuevo Hazelnut Macchiato y descubre la perfección de su sabor": Deseo, felicidad

- Emocional con producto: la marca publica un mensaje con elementos emocionales y muestra o menciona el producto. Por ejemplo: "Cappuccino, perfecto para hacerlo justo como a ti te gusta. ¿Cómo lo tomas tú?”: Felicidad.

- Emocional sin producto: una publicación con elementos emocionales pero que no muestra ni menciona el producto. Por ejemplo: "La combinación perfecta entre aroma y sabor \#pasiónporelcafé": Pasión.

- Lanzamiento con elementos emocionales: cuando hay un producto nuevo y el mensaje publicado es puramente emocional. Por ejemplo: «iEl mejor complemento para tu café ya está aquí! Nuevas \#donuts": Felicidad.

- Racional: aquellas publicaciones que muestran elementos racionales como precio, medioambiente o salud. Por ejemplo: "Recuerda que puedes traer tu propio vaso o vaso reutilizable para cuidar el medio ambiente \#mesdelatierra": Ambiente

- Promocional con elementos racionales: cuando la marca publica un mensaje promocional que habla directamente sobre el precio. Por ejemplo: "Recuerda que de 1 a 5 PM nuestros Refreshers están a mitad de precio, japrovecha y re-energízate!": Precio.

Después de identificar las categorías, separamos los comentarios en positivo y negativo, racional y neutral (aquellos que no tenían relación con la marca donde la gente comenta sobre otros temas) para cada categoría. Entre los comentarios positivos, identificamos que las personas expresan deseo mediante el uso de verbos.

Por el contrario, los comentarios que expresan pasión y amor lo hacen mediante el uso de adverbios. Estas expresiones son parte de emociones positivas secundarias, que son consecutivamente parte de la emoción primaria de la felicidad. A partir de los comentarios negativos, podemos observar expresiones relacionadas con emociones secundarias como la ira, a menudo asociadas a cuestiones racionales 
(precio, calidad, salud, promociones, responsabilidad social). Además encontramos algunos comentarios racionales que enfatizan problemas de precio, promoción, salud y responsabilidad social.

La tabla 7 contiene el porcentaje de comentarios que emergió de cada categoría. Se observó que los comentarios positivos tienen el porcentaje más alto en cada categoría, especialmente en lanzamientos con elementos emocionales. Los comentarios negativos apenas están expuestos, mientras que los racionales apenas existen, excepto cuando la marca publica un mensaje promocional con elementos racionales, como el precio.

Tabla 7. Distribución de comentarios por categoría de publicación

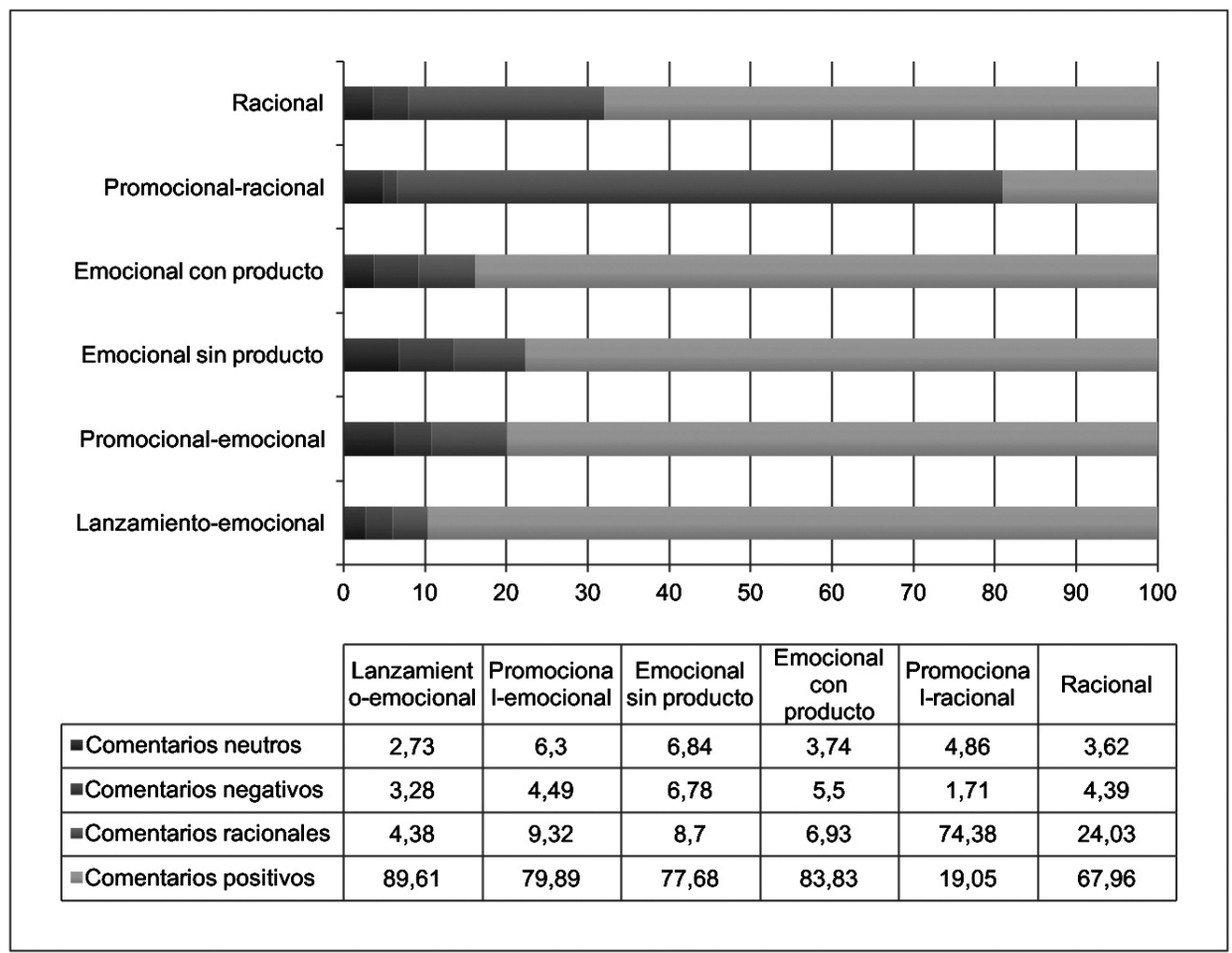

El estudio indica que la marca (Starbucks) utiliza principalmente elementos emocionales centrados en la felicidad para comunicar sus promociones. También se encontró que las categorías que incluyen promociones y lanzamientos con un elemento emocional producen el mayor número de expresiones emocionales, mientras que las publicaciones racionales generan la menor cantidad de comentarios totales.

Se confirmó que los elementos emocionales generan una mayor participación entre los miembros de una comunidad de marca, especialmente cuando hay una emoción positiva expresada a través de las emociones secundarias, como el deseo, el placer, el amor y la alegría. Además, se analizó que las emociones negativas son una forma 
importante de interacción dentro de una comunidad de marca, expresada principalmente a través de la nostalgia, la ira y la frustración, especialmente cuando hay una queja.

El análisis indica que un mensaje emocional, a pesar de ser una promoción o lanzamiento de producto, desarrolla una mayor cantidad de comentarios positivos.

Este estudio expuso que los elementos emocionales desarrollan la participación y generan un mayor interés en un tema determinado, promoviendo la comunicación dentro de una comunidad de marca en las redes sociales. También confirma que las emociones positivas se extienden más fácilmente a través de Facebook durante los períodos de vacacionales y fines de semana y durante las horas punta como el día de San Valentín, las vacaciones de primavera y el horario de verano.

De los 13043 comentarios analizados, los elementos emocionales representaron el $85,26 \%$ del total de la muestra. Dentro de los elementos emocionales observados se encontró que el $84.62 \%$ son emociones positivas, mientras que solo el 15,38\% eran negativas. Por su parte, $14,74 \%$ de los comentarios fueron elementos racionales y solo el 4,84\% fueron neutrales o temas no relacionados con la marca.

\section{Discusión y conclusiones}

\subsection{Hallazgos y aportaciones}

A fin de identificar los mensajes publicados por la marca que producen un mayor volumen de comunicación, así como la variación de las emociones expresadas por los consumidores para interactuar con otros miembros, el propósito de este estudio fue observar los elementos emocionales más representativos presentes en los mensajes. En este proyecto, se exploró la expresión de las emociones desarrolladas dentro de una comunidad de marca en línea (Starbucks México en Facebook). A través del análisis de comentarios se observó que las emociones promovían la participación y la recomendación, es decir, que las emociones generan más participación y volumen de comunicación dentro de una comunidad de marca en línea.

La investigación incluye las emociones más frecuentes expresadas por sus miembros y los temas publicados por la empresa. Percibimos que los miembros también mostraban sus emociones a través de elementos lingüísticos, como adjetivos, verbos, emoticones e imágenes (Carvalho et al., 2009; Pell et al., 2009). A menudo se observaron elementos paralingüísticos como la onomatopeya y los signos de puntuación, que daban mayor énfasis y significado a las emociones.

Las emociones son herramientas importantes que las empresas utilizan en el proceso de comunicación. Una de las ventajas de los mensajes emocionales es que a través de las comunidades de marca en línea, las empresas pueden interactuar fácilmente con los consumidores que comparten sus experiencias sobre productos o servicios en las redes sociales. Esto representa una gran oportunidad para reconocer los deseos y preferencias de los consumidores, así como para identificar las causas de su disgusto y quejas en un período de tiempo más corto.

Tomando lo anterior en consideración, las marcas pueden destacar los valores de los productos o servicios basados en aspectos emocionales para aumentar las posibilidades de ser elegidos por el consumidor. 
Proponemos así que las estrategias emocionales son más efectivas cuando la comunicación se orienta a las emociones positivas; ellas son incluso más influyentes cuando se enfocan en la felicidad. De esta manera el presente estudio proporciona pistas para la creación de estrategias de comunicación alternativas, que se vuelven cada vez más complejas, ya que las empresas deben adaptarse a los cambios de los hábitos y conductas de los consumidores.

El estudio de las emociones en una comunidad de marca en línea puede extraerse para obtener ideas sobre cómo las empresas influyen en algunos patrones de compra, que pueden explotarse de muchas maneras.

En este artículo nos centramos en lo que hace felices a las personas y en mostrar cómo los clientes quedan sorprendidos. Confirmamos que los miembros de las comunidades en línea prefieren elementos emocionales que activen las conversaciones. Esta investigación apoya que la expresión de las emociones es un elemento clave para las redes sociales en línea. El estudio de las expresiones emocionales en una comunidad de marca es especialmente importante para las empresas y otras organizaciones para mantener el vínculo con sus consumidores con un propósito a primera vista más social que económico. Además, las empresas pueden descubrir elementos negativos a considerar, brindar apoyo y retroalimentación a sus clientes.

En esta investigación confirmamos también el dominio de las emociones positivas. Se detectó que las que predominan son el deseo, la pasión, la alegría y el amor, lo que genera un mayor volumen de comunicación. Apoyamos que la pasión y el deseo son las emociones secundarias que generan más participación. También afirmamos que los mensajes positivos tienden a ser enviados por más personas, ya que la felicidad es contagiosa dentro de una comunidad de marca. Además, se observó que las emociones negativas generan una menor interacción con el consumidor y se exponían principalmente a través de la frustración y el disgusto. Se estableció que los comentarios negativos se centran más en el disgusto por algunos productos. Además, el descontento y la frustración pueden explotarse positivamente ya que son fuentes importantes de información y participación; por tanto, la participación y los comentarios proporcionan pistas para comprender algunas debilidades de la marca.

Sin embargo, la nostalgia, como parte de las emociones negativas, conduce a una experiencia positiva a través de los recuerdos. También establecimos que las publicaciones racionales generaban menor cantidad de comentarios y presentaban una cantidad considerable de elementos racionales que otro tipo de publicaciones. Los comentarios racionales se centran principalmente en la calidad del servicio, el precio y los problemas de salud. Percibimos que un mensaje emocional publicado por la marca junto con un elemento racional, produce un mayor volumen de comunicación.

Este estudio, consistente con la literatura analizada, permite hacer hincapié sobre la necesidad de favorecer una comunicación con elementos emocionales fuertes. La comunicación racional, cuyo caracter promocional resalta de manera obvia la finalidad comercial, genera menos interés. La comunicación en redes sociales, como es el caso de Facebook, debe interesarse antes que todo en el caracter justamente social de la relación con los consumidores. Las estrategias utilizadas por Starbucks, o tipos de comunicación que mejor funcionaron para mantener la atención de sus fans, consiguieron un equilibrio entre la presencia del producto o promoción y el 
carácter emocional. Si se quiere desarrollar la relación social con los consumidores, se recomienda en consecuencia favorecer las estrategias del lanzamiento-emocional y emocional con producto, ya que así se consigue un equilibrio entre la presencia de productos de la marca y refuerzo positivo de la relación con los fans.

\subsection{Limitaciones}

Algunas limitaciones detectadas en este estudio nos llevaron a encontrar cierta dificultad para distinguir si la comunicación textual fue suficiente para analizar al consumidor o si el uso de imágenes publicadas por la marca también es necesario.

Otra restricción percibida fue que ciertos elementos como signos de puntuación y palabras onomatopéyicas y jergas no son lo suficientemente claras como para distinguir la emoción. Para comprender el significado de los elementos anteriores, resultó necesario conocer el contexto y comprender el repertorio cultural utilizado en los mensajes (que creemos que se han logrado, ya que ambos codificadores eran nativos de México).

De manera similar, la ironía no fue especificamente codificada. Sin instrucciones explícitas sobre el tema, las ironías y dobles sentidos fueron directamente clasificadas en las categorías preestablecidas en fución de la comprensión de los codificadores. Las dos personas codificadoras, siendo mexicanas, se pensaría que no habría problemas de entendimiento debido a una diferencia cultural. No obstante podría ser interesante hacer una investigación teniendo en cuenta las ironías, dobles sentidos y trolling.

Una limitante más fue la complejidad para diferenciar los comentarios racionales y negativos, ya que los consumidores mostraron sus emociones negativas cuando apareció un problema racional como el precio, la calidad y el servicio.

Finalmente, otra de las limitaciones significativas enfrentadas sucedió al ejecutar la doble codificación (Kappa). Los resultados mostraron una diferencia significativa entre los codificadores en la emoción de la alegría. Una posible razón para este fenómeno podría ser la similitud en la forma en que las personas expresan su pasión y deseo. Dado que estas emociones contienen una connotación positiva, las personas las toman como sinónimos de alegría o felicidad.

\subsection{Futuras investigaciones}

De manera general, sería interesante, retomar o profundizar ciertos puntos: analizar las emociones por separado ya que se observó que cada una produce un nivel diferente de participación y volumen de comunicación pero no se estudió la intensidad de aquellas; profundizar el análisis de la alegría ya que su codificación resultó menos convergente que las demás; profundizar el estudio de la nostalgia ya que si se codifica como una emoción negativa, el sentimiento y los efectos que acaba generando parecen ser positivos; hacer entrevistas o grupos focales (focus groups) para entender por qué los elementos racionales generan menos participación; estudiar las imágenes publicadas para relacionarlas con emociones; retomar y/o extender la investigación tomando en cuenta ironías, dobles sentidos y trolling. 


\section{Referencias}

Aguado-Guadalupe, G. (2017). Repercusión de las métricas de audiencia online en la comercialización publicitaria del producto informativo. Razón y Palabra, 21(97).

Ashforth, B. E., \& Mael, F. (1989). Social identity theory and the organization. Academy of management review, 14(1), 20-39.

Asociación de internet.MX. (2018). 14 Estudio sobre los Hábitos de los Usuarios de Internet en México 2018 (p. 27). México: Asociación de internet.MX. Recuperado de https://www. asociaciondeinternet.mx/es/component/remository/Habitos-de-Internet/14-Estudio-sobre-los-Habitos-de-los-usuarios-de-Internet-en-Mexico-2018/lang,es-es/?Itemid=

Auladell, F. V. (2016). La actividad publicitaria: entre el simulacro y la utopía. Universitas Humanística, (81), 149-176.

Barroso Morales, M. Á. (2011). "Hoy me siento contento, feliz día a todos”. El escaparate virtual de las emociones posmodernas llamado Facebook. En M. P. Bianchi \& L. R. Sandoval (Eds.). Presentado en Jornadas Patagónicas de estudios sociales sobre Internet y tecnologías de la comunicación, Comodoro Rivadavia: Universidad Nacional de la Patagonia San Juan Bosco. Recuperado de http://tecnologiaycultura.com.ar/actas2011/doc/m6barroso.pdf

Baun, D., \& Gröppel-Klein, A. (2003). Joy and surprise as guides to a better understanding of impulse buying behaviour. European Advances in Consumer Research, 6, 290-299.

Belk, R. W., Ger, G., \& Askegaard, S. (2003). The Fire of Desire: A Multisited Inquiry into Consumer Passion. Journal of Consumer Research, 30(3), 326-351. https://doi.org/10.1086/378613

Berelson, B. (1952). Content analysis in communication research. New York, NY, US: Free Press.

Berger, J., \& Milkman, K. L. (2012). What Makes Online Content Viral? Journal of Marketing Research, 49(2), 192-205. https://doi.org/10.1509/jmr.10.0353

Brady, W. J., Wills, J. A., Jost, J. T., Tucker, J. A., \& Van Bavel, J. J. (2017). Emotion shapes the diffusion of moralized content in social networks. Proceedings of the National Academy of Sciences, 114(28), 7313-7318. https://doi.org/10.1073/pnas.1618923114

Buck, R. (2006). The evolutionary bases of social and moral emotions: Dominance, submission, and true love. En The evolution of the social mind: Evolutionary psychology and social cognition. Sidney, Australia: SSSP. Recuperado de http://www.sydneysymposium.unsw.edu.au/2006/ DraftChapters.htm

Burgoon, J. K., Guerrero, L. K., \& Floyd, K. (2010). Nonverbal communication. Boston: Allyn \& Bacon.

Carvalho, P., Sarmento, L., Silva, M. J., \& de Oliveira, E. (2009). Clues for detecting irony in user-generated contents: oh...!! it's "so easy" ;-). En Proceeding of the 1st international CIKM workshop on Topic-sentiment analysis for mass opinion - TSA '09 (p. 53). Hong Kong, China: ACM Press. https://doi.org/10.1145/1651461.1651471

Codina, J. N. B., Rodríguez, P. G., \& Cadena, P. V. (2017). La economía de la experiencia y el marketing emocional: estrategias contemporáneas de comercialización. Daena: International Journal of Good Conscience, 12(2), 159-170.

Cohen, E. L. (2014). What makes good games go viral? The role of technology use, efficacy, emotion and enjoyment in players' decision to share a prosocial digital game. Computers in Human Behavior, 33, 321-329. https://doi.org/10.1016/j.chb.2013.07.013

Cordelier, B., \& Turcin, K. (2005). Utilisations du lien social sur l'Internet comme élément fidélisant à une marque. Les exemples de Coca-Cola et d'ESP. Communication $\mathcal{E}$ Organisation, (27), 45-56.

Coviello, L., Sohn, Y., Kramer, A. D. I., Marlow, C., Franceschetti, M., Christakis, N. A., \& Fowler, J. H. (2014). Detecting Emotional Contagion in Massive Social Networks. PLoS ONE, 9(3), e90315. https://doi.org/10.1371/journal.pone.0090315

Dessart, L., Veloutsou, C., \& Morgan-Thomas, A. (2015). Consumer engagement in online brand communities: a social media perspective. Journal of Product $\mathcal{E}$ Brand Management, 24(1), 28 42. https://doi.org/10.1108/JPBM-06-2014-0635

Evans, D. (2002). Emotion: the science of sentiment. Oxford: Oxford Univ. Press. 
Expansión. (2017, septiembre 5). Los 15 datos de los 15 años de Starbucks en México. Recuperado el 9 de enero de 2019, de https://expansion.mx/empresas/2017/09/04/los-15-datos-delos-15-anos-de-starbucks-en-mexico

Firat, A. F., \& Venkatesh, A. (1995). Liberatory Postmodernism and the Reenchantment of Consumption. Journal of Consumer Research, 22, 239-267.

Fowler, J. H., \& Christakis, N. A. (2008). Dynamic spread of happiness in a large social network: longitudinal analysis over 20 years in the Framingham Heart Study. BMJ, 337(dec04 2), a2338-a2338. https://doi.org/10.1136/bmj.a2338

Frederickson, B. (2017). Biological Underpinnings of Positive Emotions and Purpose. The social psychology of good life. Sidney, Australia: SSSP. Recuperado de http://www.sydneysymposium.unsw.edu.au/2017/chapters/chapters.htm

Fu, P.-W., Wu, C.-C., \& Cho, Y.-J. (2017). What makes users share content on facebook? Compatibility among psychological incentive, social capital focus, and content type. Computers in Human Behavior, 67, 23-32. https://doi.org/10.1016/j.chb.2016.10.010

Goulding, C. (2013). Learning to be tribal: facilitating the formation of consumer tribes. European Journal of Marketing, 47(5), 813-832. https://doi.org/10.1108/03090561311306886

Goulding, C., Shankar, A., Elliott, R., \& Canniford, R. (2009). The Marketplace Management of Illicit Pleasure. Journal of Consumer Research, 35(5), 759-771. https://doi.org/10.1086/592946

Guerreiro, J., Rita, P., \& Trigueiros, D. (2015). Attention, emotions and cause-related marketing effectiveness. European Journal of Marketing, 49(11/12), 1728-1750. https://doi.org/10.1108/ EJM-09-2014-0543

Hochschild, A. R. (1979). Emotion Work, Feeling Rules, and Social Structure. American Journal of Sociology, 85(3), 551-575. https://doi.org/10.1086/227049

Hollebeek, L. D., Juric, B., \& Tang, W. (2017). Virtual brand community engagement practices: a refined typology and model. Journal of Services Marketing, 31(3), 204-217. https://doi. org/10.1108/JSM-01-2016-0006

Huang, M.-H. (2001). The Theory of Emotions in Marketing. Journal of Business and Psychology, 16(2), 239-247.

Hudson, S., Roth, M. S., Madden, T. J., \& Hudson, R. (2015). The effects of social media on emotions, brand relationship quality, and word of mouth: An empirical study of music festival attendees. Tourism Management, 47, 68-76. https://doi.org/10.1016/j.tourman.2014.09.001

Hupp, O., Gröppel-Klein, A., Dieckmann, A., Broeckelmann, P., \& Walter, K. (2008). Beyond verbal scales: Measurement of emotions in advertising effectiveness research. Yearbook of Marketing and Consumer Research, 6(4), 72-99.

Illouz, E. (2007). Cold intimacies: the making of emotional capitalism. Cambridge, UK ; Malden, MA: Polity Press.

Kassarjian, H. H. (1977). Content Analysis in Consumer Research. Journal of Consumer Research, 4(1), 8. https://doi.org/10.1086/208674

Khuong, M. N., \& Tram, V. N. B. (2015). The Effects of Emotional Marketing on Consumer Product Perception, Brand Awareness and Purchase Decision - A Study in Ho Chi Minh City, Vietnam. Journal of Economics, Business and Management, 3(5), 524-530. https://doi. org/10.7763/JOEBM.2015.V3.240

Kolbe, R., \& Albanese, P. (1997). The Functional Integration of Sole-Male Images into Magazine Advertisements. Sex Roles, 36(11-12), 813-836. https://doi.org/10.1023/A:1025683324423

Kramer, A. D. I., Guillory, J. E., \& Hancock, J. T. (2014). Experimental evidence of massive-scale emotional contagion through social networks. Proceedings of the National Academy of Sciences, 111(24), 8788-8790. https://doi.org/10.1073/pnas.1320040111

Le Breton, D. (2012). Por una antropología de las emociones. Revista Latinoamericana de Estudios sobre Cuerpos, Emociones y Sociedad, 69-79.

Liu, B. (2012). Sentiment Analysis and Opinion Mining. Synthesis Lectures on Human Language Technologies, 5(1), 1-167. https://doi.org/10.2200/S00416ED1V01Y201204HLT016 
Manchanda, P., Packard, G., \& Pattabhiramaiah, A. (2015). Social Dollars: The Economic Impact of Customer Participation in a Firm-Sponsored Online Customer Community. Marketing Science, 34(3), 367-387. https://doi.org/10.1287/mksc.2014.0890

Muñiz, A. M., \& O'Guinn, T. C. (2001). Brand Community. Journal of Consumer Research, 27(4), 412 432. https://doi.org/10.1086/319618

Nabi, R. L. (2017). Emotion in Media Persuasion. En P. Rössler, C. A. Hoffner, \& L. van Zoonen (Eds.), The International Encyclopedia of Media Effects (pp. 1-8). Hoboken, NJ, USA: John Wiley \& Sons, Inc. https://doi.org/10.1002/9781118783764.wbieme0161

Neuendorf, K. A. (2017). The content analysis guidebook (Second edition). Los Angeles: SAGE.

Pell, M. D., Monetta, L., Paulmann, S., \& Kotz, S. A. (2009). Recognizing Emotions in a Foreign Language. Journal of Nonverbal Behavior, 33(2), 107-120. https://doi.org/10.1007/s10919-0080065-7

Plutchik, R. (1994). The psychology and biology of emotion (1st ed). New York, NY: HarperCollinsCollegePublishers.

Plutchik, R. (2001). The Nature of Emotions: Human emotions have deep evolutionary roots, a fact that may explain their complexity and provide tools for clinical practice. American Scientist, 89(4), 344-350.

Ribes, X., Monclús, B., Gutiérrez García, M., \& Martí, J. M. (2017). Aplicaciones móviles radiofónicas: adaptando las especificidades de los dispositivos avanzados a la distribución de los contenidos sonoros. Revista de la Asociación Española de Investigación de la Comunicación; Vol. 4 Núm. 7 (2017): El futuro de la radio. Recuperado de http://www.revistaeic.eu/index.php/raeic/ article/view?path=

Roberts, K. (2005). Lovemarks: the future beyond brands (2nd ed., Expanded ed). New York, NY: PowerHouse Books.

Schau, H. J., Muñiz, A. M., \& Arnould, E. J. (2009). How Brand Community Practices Create Value. Journal of Marketing, 73(5), 30-51. https://doi.org/10.1509/jmkg.73.5.30

Schembri, S., \& Latimer, L. (2016). Online brand communities: constructing and co-constructing brand culture. Journal of Marketing Management, 32(7-8), 628-651. https://doi.or$\mathrm{g} / 10.1080 / 0267257 X .2015 .1117518$

Scolari, C. (2013). Hipermediaciones elementos para una teoría de la comunicación digital interactiva. Barcelona: Editorial Gedisa.

Segarra-Saavedra, J., \& Tur-Viñes, V. (2017). Creatividad publicitaria: marcas vs prosumer amateur. La viralidad del spot de Eugen Merher para Adidas.

Shankar, A., Elliott, R., \& Fitchett, J. (2009). Identity, consumption and narratives of socialization. Marketing Theory, 9(1), 75-94. https://doi.org/10.1177/1470593108100062

Thompson, C. J., Rindfleisch, A., \& Arsel, Z. (2006). Emotional Branding and the Strategic Value of the Doppelgänger Brand Image. Journal of Marketing, 70(1), 50-64. https://doi. org/10.2307/30162072

Vincent, J., \& Fortunati, L. (Eds.). (2009). Electronic emotion: the mediation of emotion via information and communication technologies. Oxford ; New York: Peter Lang.

Weiger, W. H., Hammerschmidt, M., \& Wetzel, H. A. (2018). Don't You Dare Push Me: How Persuasive Social Media Tactics Shape Customer Engagement. Journal of the Association for Consumer Research, 3(3), 364-378. https://doi.org/10.1086/698713

Yadollahi, A., Shahraki, A. G., \& Zaiane, O. R. (2017). Current State of Text Sentiment Analysis from Opinion to Emotion Mining. ACM Computing Surveys, 50(2), 1-33. https://doi. org/10.1145/3057270

Yu, J. (2014). We Look for Social, Not Promotion: Brand Post Strategy, Consumer Emotions, and Engagement - A Case Study of the Facebook Brand Pages. GSTF Journal on Mobile Communications, Networking and Applications (JMC) Vol.1 No.2, 1(2), 32-41. https://doi. org/10.5176/2335-6618_1.2.17 